\title{
Research of Job Scheduling With Cloud Based On Trust Mechanism And SFLA
}

\author{
Xiaolan Xie ${ }^{1}$, Ruikun Liu ${ }^{1}$, Guoqing Zhou ${ }^{1}$ and Jinsheng $\mathrm{Ni}^{1}$ \\ 'Institute of Information Science and Engineering, Guilin University of \\ Technology, Guilin, China \\ xie_xiao_lan@foxmail.com
}

\begin{abstract}
Job scheduling under the cloud circumstance is the key techniques to impact on frequency of cloud calculation. Trust relationship is an important factor of cloud calculation and job scheduling. In order to satisfy the requirement of quality of cloud calculation service for job scheduling, this article introduced the term of trust mechanism and apply it to cloud calculation and job scheduling. It uses altered SFLA as scheduling strategy, and brings forward a model of job scheduling with cloud circumstance which is based on trust mechanism and SFLA. The simulation results showed that this algorithm with faster convergence speed can increase the trust benefit and accomplish job scheduling task well.
\end{abstract}

Keywords: cloud computing task scheduling trust mechanism SFLA

\section{Introduction}

Cloud computing is an outstanding calculation method witch support service demand services, the Internet can expand resources as a service delivery to customers.It can see the extensible resource on the Internet as a service delivery to customers. Scheduling in cloud computing will affect the effect of cloud computing, so scheduling is the key link to improve the quality of service of cloud computing[1].Most of the traditional scheduling algorithm regards reduce scheduling time as the main target, Such as the classical Greedy, Min-Min, Max-min. In addition, there are some scheduling algorithm based on artificial intelligence such as ant colony algorithm, genetic algorithm.

However, the main goal of cloud computing is to provide on-demand service for users. The traditional scheduling algorithm can not satisfy the Qos requirements of users, most of them ignore the reliability and trust relation of cloud resource service. Therefore, job scheduling with cloud based on trust mechanism becomes increasingly important. Task scheduling algorithm based on trust mechanism needs trust specific model . In order to prevent defects of separation of trust mechanism and scheduling mechanism on grid resource management, Document[2] proposeds the grid job scheduling algorithm based on trust relationship which are built on Separation of trust mechanism and scheduling mechanism. The literature[3] analysis of the potential factors that may affect the normal execution of tasks, And proposed a grid task scheduling framework and related algorithms which is driven by trust QoS.The literature[4] divided scheduling into two levels, the first level is the overall scheduling which takes the users as the unit,the second is micro scheduling between tasks and available resources .And they proposed a scheduling

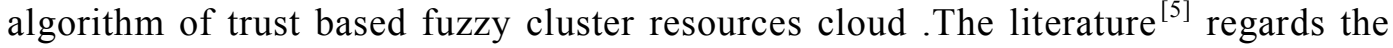
trust relationship as a safety measure and integrate it into task scheduling strategy in grid environment, then design a scheduling algorithm named Trustbased Min-min. In addition, the literature ${ }^{[6]}$ uses trust relationship in human society for reference from and puts it into the trust mechanism. It removes the task on error node Dynamically, and propose a dynamic task scheduling model based on trust 
mechanism. Based on the above analysis, this paper puts trust mechanism into the scheduling on cloud environment, and using the shuffled frog leaping algorithm, in order to better meet the needs of trust requirements and efficiency of tasks.

\subsection{Task Scheduling Model based on Trust Mechanism}

In the scheduling, in order to maintain and manage the trust degree of resource nodes in cloud environment, trust mechanism is introduced in the traditional framework of resource scheduling. Scheduling model as shown in figure 1.

Based on this model, the task scheduling process is as follows:

(1) The user submits the trust information of element task and the element task to "submitting center" in the cloud environment;

(2) After the task is referred, "submitting center" will save it to the "task task queue", then "Qos task demand query module" queries available resources in cloud according to the task Qos requirements, and returns the query results to the "Qos query module";

(3) "Qos query module" pre selects a subset of available resources according to query results;

(4) Task scheduling system computing trust degree of each resources in available resources subset according to the trust mechanism;

(5) Task scheduling system combines trust information of each resources, Returns the set of resources which meet users requirement,and schedule the collection of resources with SFLA.The task are assigned to the different resource nodes according to the scheduling results until the tasks finish.

\section{Problem Description}

\subsection{The Trust Relationship Model}

This paper defines trust model on cloud based on the difference between the trust domain. The trust model contains the following elements: $\mathrm{TM}=(\mathrm{I}, \mathrm{J}, \mathrm{K}, \mathrm{t})$.I is the credit side, $\mathrm{J}$ is a trusted party, $\mathrm{K}$ is recommended, $\mathrm{K}=\{\mathrm{K} 1, \mathrm{~K} 2, \mathrm{Kn}\}, \mathrm{I}, \mathrm{J}, \mathrm{K}$ are entity in the cloud computing environment . $\mathrm{T}$ is the time constrained of trust relationships.

Definition 1 Trust At the evaluation process of the credit side to the credibility of the trusted party's behaviour, the Trust is a subjective evaluation to the trust party,which is highly dependent on the credit side's experience and knowledge background.

Definition 2 Trust Degree This is a evaluation of the trust level of a trusted party, trust degree can be continuous or discrete, denoted as TV (I, J), this represents the trust degree of two nodes I and J. 


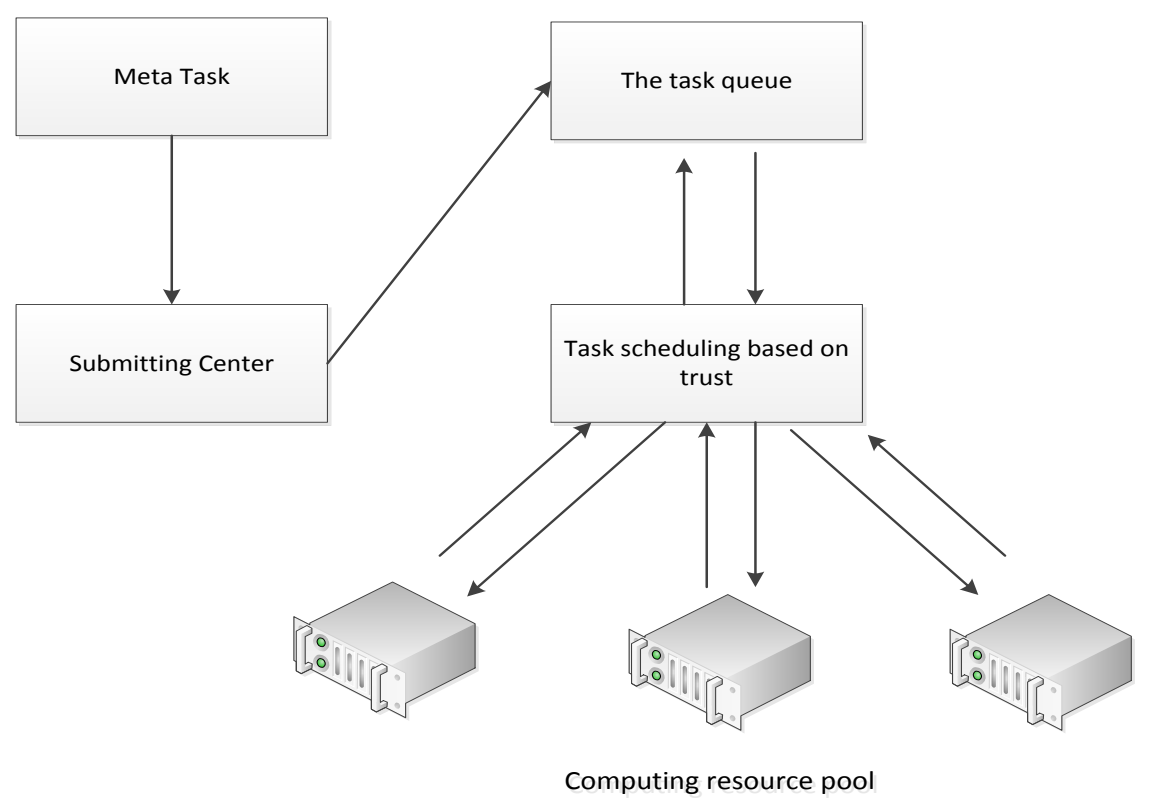

Figure 1. Task Scheduling Model based on Trust Mechanism

Definition 3 Direct Trust This means the trusted party and the credit side complete the direct interaction, They both exist direct trust relationship. Denoted as DTV (I, J), this represents the direct trust degree of I to $\mathrm{J}$.

Definition 4 Recommendation Trust This means that before the direct interaction of the trusted party and the credit side, The trust relationship established by recommendation of other entities. Denoted as RTV (B, J), this represents the recommendation trust degree of B to J.

Definition 5 Risk This reflects the unreliable degree of a trusted party within the recent period of time, it is obtained through the direct interaction of the credit side and the trusted party. The model reaction through the interaction of failure rate. The model reflects it through the failure rate of the interaction.

\subsection{Calculation of Trust Degree}

(1) Calculation of direct trust degree DTV(I,J,t) of I to J in a moment t,it is shown in the formula

$$
\operatorname{DTV}(\mathrm{I}, \mathrm{J}, \mathrm{t})=\gamma \operatorname{DTV}\left(\mathrm{I}, \mathrm{J}, \mathrm{t}_{1}\right) \mathrm{A}\left(\mathrm{I}, \mathrm{J}, \mathrm{t}-\mathrm{t}_{1}\right)-(1-\gamma) \mathrm{R}\left(\mathrm{J}, \mathrm{t}-\mathrm{t}_{1}\right)
$$

In the formula, $\gamma$ is weighted factor, value between $[0,1]$, it is used to adjust trust concerns time range. DTV (I, J, T1) is the direct trust interactive value of $\mathrm{I}, \mathrm{J}$ at the moment of $t_{1}, A\left(I, J, t-t_{1}\right)$ is the time decay function, It is calculated as $A\left(I, J, t-t_{1}\right)=$ $\frac{1}{1+(t-t 1) / V}$

(2) Calculation of Recommendation Trust degree RTV $(B, J, t)$ of $K$ to $J$ in a moment $t$,it is shown in the formula (2).

$$
\operatorname{RTV}(\mathrm{B}, \mathrm{J}, \mathrm{t})=\frac{\rho(n) \times \sum_{k=1}^{n} \operatorname{RTV}\left(\mathrm{B}_{k}, \mathrm{~J}, \mathrm{t}_{1}\right) \times \gamma\left(t-t_{1}\right)}{n}
$$

In the formula, $\gamma$ ibid (1), $\mathrm{n}$ is the number of cloud trust domain. In order to resist some malicious entity slander attack, Using a function $\rho(n)$ to regulate the Influence of the 
number of recommended entity to the recommendation trust degree. This function reflects the evaluation approaches to the the average of entity's evaluation with the increasing of number of evaluation of node service quality. Here take $\rho(n)=e^{-\frac{1}{n}}$.

(3) In summary, Calculation of comprehensive trust degree TV $(\mathrm{I}, \mathrm{J}, \mathrm{t})$ of I to $\mathrm{J}$ in a moment t,it is shown in the formula (3).

$$
\operatorname{TV}(\mathrm{I}, \mathrm{J}, \mathrm{t})=\alpha \mathrm{DTV}(\mathrm{I}, \mathrm{J}, \mathrm{t})+\beta \mathrm{RTV}(\mathrm{B}, \mathrm{J}, \mathrm{t})
$$

determining the value of $\alpha, \beta$ according to the specific environment of cloud to , They value between $[0,1]$, And $\alpha+\beta=1$.

\section{Design of Shuffled Frog Leaping Algorithm}

Shuffled frog leaping algorithm is a kind of collaborative search method based on the population natural which is produced by enlightenment of natural biological imitation[7].SFLA is divided into the local search and global information exchange. Through the combination of the two,Selecting the best individual in the local,Then using the shuffle strategy,And global exchanging the local information.Continue until it meets constraint conditions.

(1) Meme Code

Coding is important, according to the characteristics of the tasks and resources in cloud environment, this paper adopts real coding, The length of meme code is equal to the number of task, The code' $\mathrm{s}$ information is equal to the number of resources

(2)Initializing total group level of fitness function

Initial population is generated by Min-Min algorithm.

The fitness function formula is shown in formula (4):

$$
\text { Fitness } f=\frac{\mathrm{TV}(\mathrm{I}, \mathrm{J}, \mathrm{t})}{\text { MakeSpan }}
$$

In the formula MakeSpan is the time span of task scheduling. The only trust value TV (I, J, t) can be calculated By formula (3). It can be obtained By the formula (4) that the smaller task scheduling time span is, and the greater the trust degree is, the greater the value of the fitness is.

\section{(3)Local search}

Doing following operations to all ethnic groups periodically within default local searching times.

1) Local optimal solution $D_{b}$ learning approach to Ethnic optimal solution $D_{g}$. The learning process consists of three steps: First, trying to move closer to the $\mathrm{D}_{\mathrm{g}}$. Second, Searching within the $\mathrm{D}_{\mathrm{g}}$ 's neighborhood. Third, Self neighborhood searching. $\mathrm{D}_{\mathrm{b}}$ updates according to the following formula.

$$
\begin{aligned}
& d=\min \left\{\operatorname{INT}\left[\left(D_{g}-D_{b}\right)\right], d_{\text {max }}\right\} \\
& \tilde{D}_{b}=D_{b}+d
\end{aligned}
$$

Where $d$ is the $D_{b}$ 's step, $d_{\max }$ is the maximum step size.

2)Through optimize the direction of search of $D_{b}$, The worst solution of ethnic group $D_{w}$ drives the ethnic group to the extreme point. The process can be divided into two stages: First, trying to learn to approach to $D_{b}$. Second, Increasing the diversity of population through self mutations. $\mathrm{D}_{\mathrm{w}}$ updates according to the following formula.

$$
d=w d+\operatorname{rand}() \times\left(D_{b}-D_{w}\right)
$$




$$
\begin{aligned}
& w=w_{\text {min }}+\left(w_{\text {max }}-w_{\text {min }}\right) \frac{I-i}{I} \\
& \tilde{D_{w}}=D_{w}+d
\end{aligned}
$$

Where $d$ is the $D_{w}$ 's step, $\operatorname{rand}() \in(0,1) ; d \in\left[d_{\text {max }}, d_{\text {min }}\right]$, d' is the earlier step, w is the attribute weight coefficient. $\mathrm{w}_{\max }$ and $\mathrm{w}_{\min }$ are the starting value and the end value of the attribute weight coefficient. When $\mathrm{w}$ is large, the ability of global search is higher. Conversely, When w is more small, the ability of local search is higher. $\mathrm{i}$ is the current number of iterations. I is the total number of iterations.

(4) Process of population shuffle

After a series of evolution of populations, Sharing information of each individual of populations. Calculating the fitness of each individual. And sorting in descending order. Then determine the $\mathrm{D}_{\mathrm{g}}$.

Figure 2 shows the flowchart of scheduling algorithm .

\section{The Results of the Simulation Experiment and Analysis}

Simulation experiments were carried out in the MyEclipse environment. Using the cloud environment simulation tool CloudSim to complete it. The article makes CloudSim be a foundation and extended class Cloudlet etc. So that it can schedule the workflow better by using shuffled frog leaping algorithm, which can support the trust mechanism. In order to show the superiority of the algorithm, We implemented TBSFLA (Trust-based Shuffled Frog Leaping Algorithm), TDMin-Min (Trust-based Min-Min) and GA (Generation Algorithm) in the simulation platform for comparison.

This paper uses The average value of the trust benefit to evaluate above algorithms. The average value of the trust benefit is acquired by the successful scheduling of task. The trust service of the is algorithm better when Average trust benefit value is higher. Setting the variable $\mathrm{Vq}[1,4]$ to control the the trust relationship between Jobs and the resources ${ }^{[8]}$. The pairs of jobs and the resources with strong trusting relationship are more when $\mathrm{Vq}$ is bigger; Generating a random number between $[0,1]$, If the number is less than 0.25 , That Job and resource have a strong trusting relationship. If the number is less than 0.5 , That Job and resource have a weak trusting relationship. Otherwise, they have no trust relationship.In this experiment, The set consists of 50 250 independent operations. The population size Pop is 100, the number of resources is 20 .The experimental results are shown in Figure 3 and Figure 4.

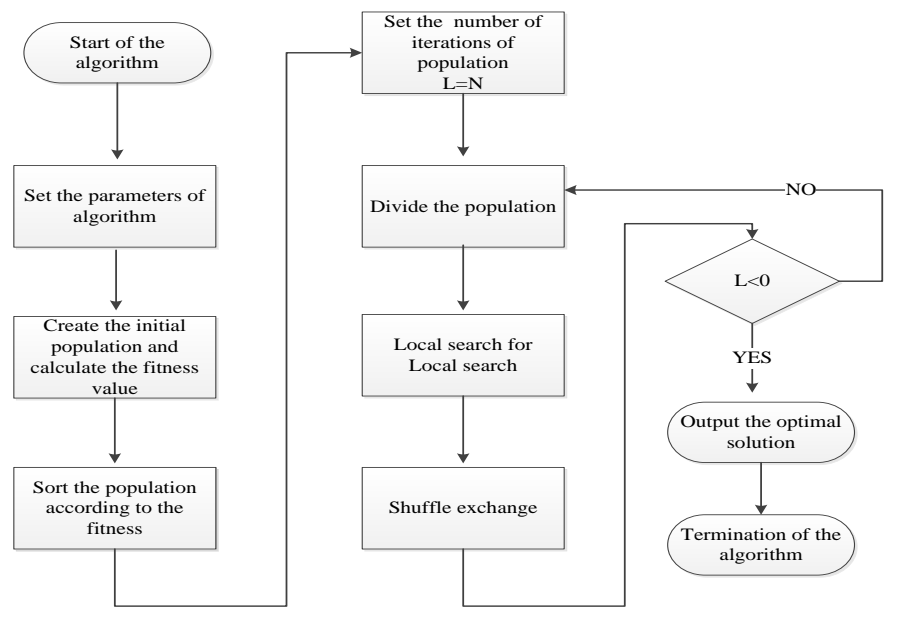

Figure 2. Flow Chart of Shuffled Frog Leaping Algorithm 


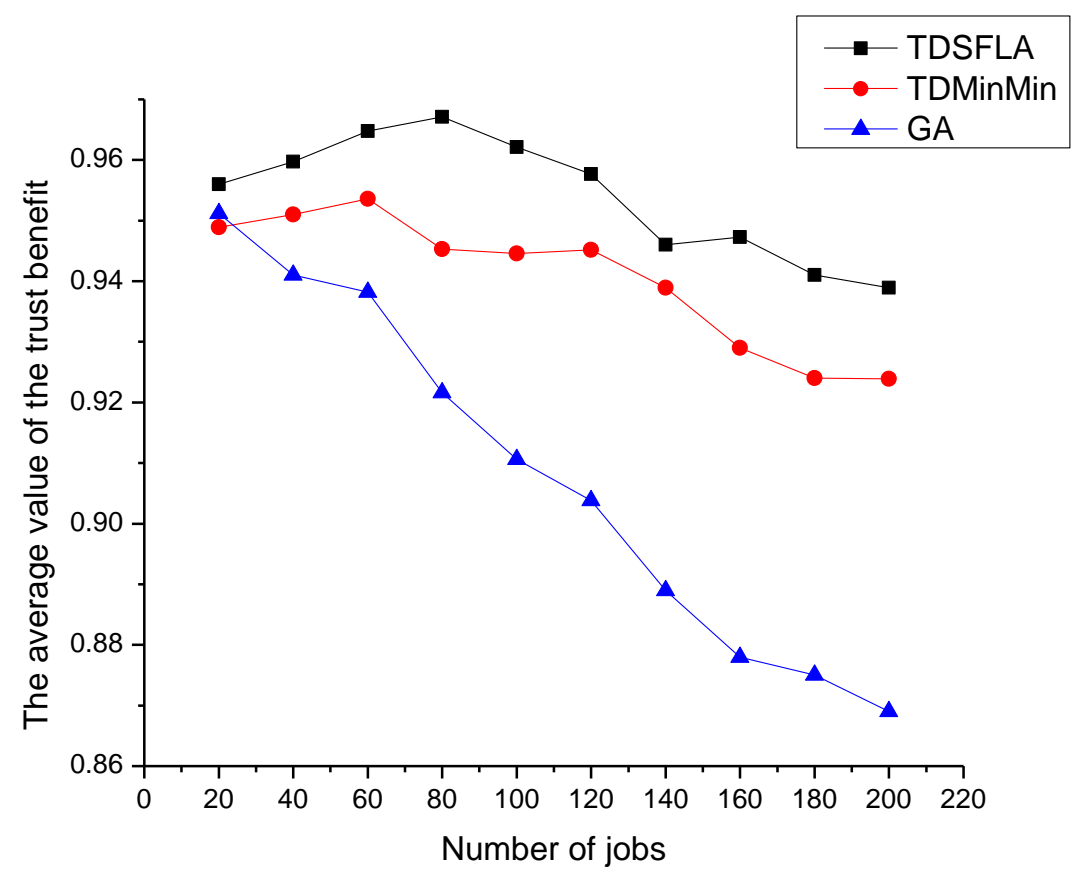

Figure 3. $\mathrm{Vq}=3$, the Average Trust Benefit Value Changes with the Number of Tasks

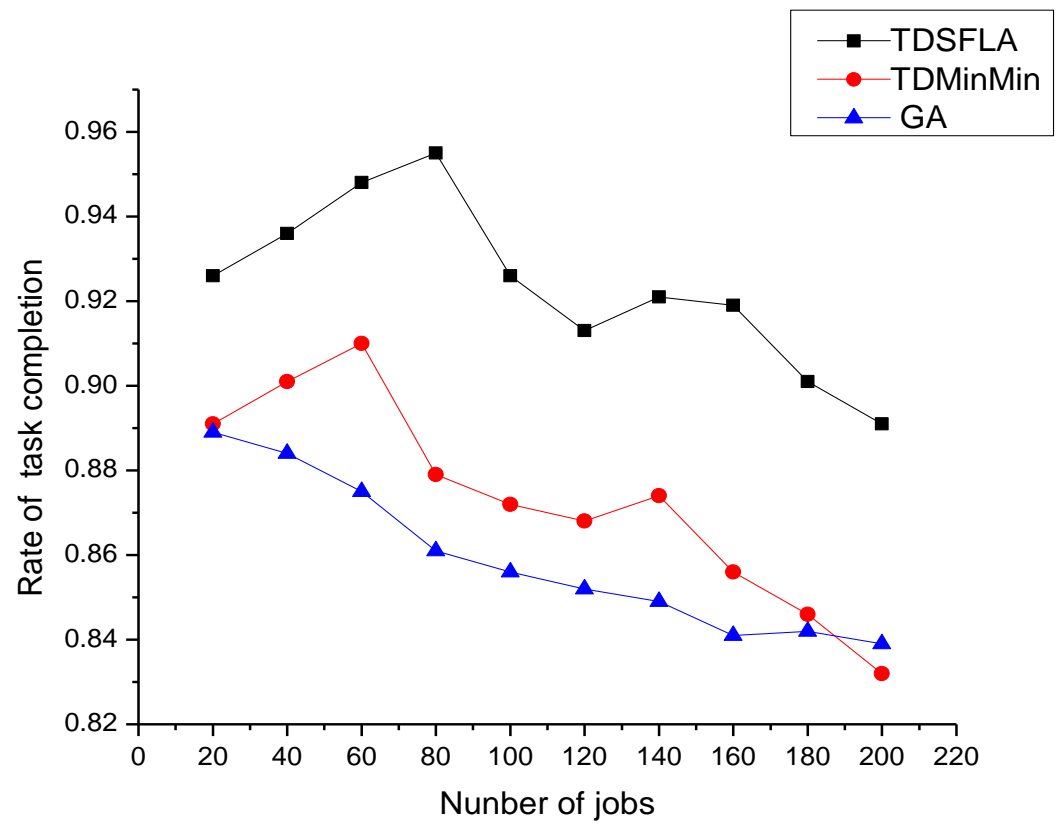

Figure 4. $\mathrm{Vq}=3$, the Rate of Task Completion Changes with the Number of Tasks

It can be seen from Figure 3, when $\mathrm{Vq}=3$, The average trust benefit value of TDSFLA and TDMinMin is better than GA by introducing the trust mechanism. While The average trust benefit of TDSFLA is slightly better than that of TDMinMin. Figure 4 shows the TDSFLA can obtain the highest rate of task completion in the circumstances.

By the experiment, the TDSFLA can obtain good average trust benefit, and can obtain 
higher task completion rate, Thus improving the scheduling quality.

\section{Summary}

This paper presents a scheduling model based on trust mechanism in cloud environment. Introduces the trust mechanism, and combines the shuffled frog leaping algorithm to schedule the the job in cloud environment. And makes comparison of Min-Min algorithm based on the trust mechanism and the traditional genetic algorithm by performing experiments. Experimental results show that the model can effectively improve the average trust benefit and successful rate of Job scheduling, and then better meet the Qos requirements of users.

Future work will improve the process of trust evaluation, and the more factors which affect service reliability will be taken into account.

\section{Acknowledgements}

This research work was supported by National High Technology Research and Development Program 863 under Grant No. 2013AA12A402, Natural Science Foundation of Guangxi Provincial under Grant No. 2013GXNSFAA019349, Gui Gongxin Dianruan under Grant No.[2013]259 and Guilin science and technology projects under Grant No. GuiKeGong 20140103-16.

\section{References}

[1] W. Yuqi and Z. Guosun, "Trend Analysis for Scheduling Algorithm in Cloud Computing", Microelectronics and Computer, Microelectronics and Computer, vol. 29, no. 9, (2012), pp. 1000-7180.

[2] Z. Weizhe and F. Binxing, "A Trust-Qos Enhanced Grid Service Scheduling", Chinese Journal of Computers, vol. 29, no. 7, (2006), pp. 1157-1166.

[3] K. Li, Y. He and X. Liu, "Security-driven scheduling algorithms based on eigentrust in grid", Proceedings of the 6th International Conference of Parallel and Distributed Computing Applications and Technologies, (2005); Denver, USA.

[4] W. J. Li, "Research on Trust-based Cloud Service Management Model and Scheduling Algorithms", ZheJiang: Zhejiang University, (2012).

[5] Y. Li and G. Huang, "Task Scheduling Algorithm in Compute Grid Based on Trust”, Journal of Beihua University, vol. 14, no. 2, (2013), pp. 1099-4822.

[6] B. Huang, "Trust mechanism-based dynamic task scheduling in Grid Computing", Computer Applications, vol. 26, no. 1, (2010), pp. 1001-9081.

[7] Y. Luan, "Development status of Shuffled frog leaping algorithm and its Research", Da Zhong Keji, vol. 1, (2009), pp. 24-25.

[8] F. Azzedin, "Muthucumaru Maheswaran, Towards Trust-Aware Resource Management in Grid Computing System", Proceedings of the 2nd IEEE/ACM International Symposium on Cluster Computing and the Gird, (2002).

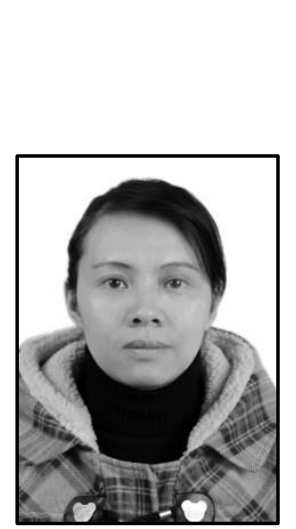

\section{Authors}

Xiaolan Xie. Female, $\mathrm{PhD}$, Professor, School of information science and engineering, Guilin University of Technology, research direction: Cloud computing, Grid computing,Intelligent decision system. 


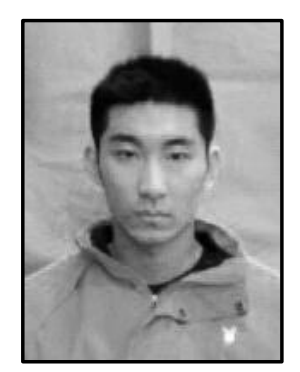

Ruikun Liu. Male, Master, School of information science and engineering, Guilin University of Technology, research direction: Cloud computing.

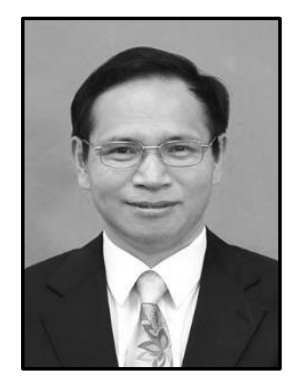

Guoqing Zhou. Male, PhD, China "The Recruitment Program of Global Experts" scholar, Professor of Guilin University of Technology.

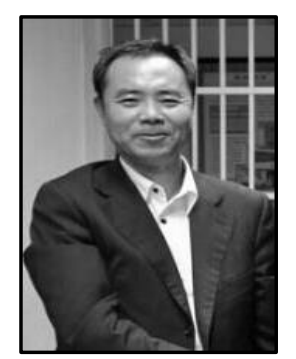

Jinshan Ni. Male, The guest professor of Guilin University of Technology, Chairman of Beijing Oriental Titan Corp. 